\title{
NULL CONTROLLABILITY OF THE SEMILINEAR HEAT EQUATION
}

\author{
E. FERNÁNDEZ-CARA
}

\begin{abstract}
This paper is concerned with the null controllability of systems governed by semilinear parabolic equations. The control is exerted either on a small subdomain or on a portion of the boundary. We prove that the system is null controllable when the nonlinear term $f(s)$ grows slower than $s \cdot \log |s|$ as $|s| \rightarrow+\infty$.
\end{abstract}

\section{INTRODUCTION}

Assume a bounded and connected open set $\Omega \subset \mathbb{R}^{N}$ with regular boundary $\partial \Omega$, a nonempty open subset $\mathcal{O} \subset \Omega$ and a positive number $T$ are given.

We will use the following notation: $Q=\Omega \times(0, T), \Sigma=\partial \Omega \times(0, T)$, $H=L^{2}(\Omega) ;|$.$| and (.,$.$) will stand for the usual norm and scalar product in$ $H$, respectively; $C$ will denote a generic constant.

In this paper, we consider systems of the form

$$
\left\{\begin{array}{l}
\partial_{t} y-\Delta y+f(y)=1_{\mathcal{O}} v \text { in } Q, \\
y=0 \text { on } \Sigma, \\
y(0)=y_{0},
\end{array}\right.
$$

where $y_{0} \in H$ and $v \in L^{2}(0, T ; H)$ are given. Here, $1_{\mathcal{O}}$ is the characteristic function of the set $\mathcal{O}$ and it is assumed that $f: \mathbb{R} \mapsto \mathbb{R}$ is locally Lipschitzcontinuous and satisfies $f(0)=0$.

It will be said that (1.1) is null controllable if there exists a control $v \in$ $L^{2}(0, T ; H)$ such that the corresponding initial-boundary problem possesses a solution $y \in C^{0}(0, T ; H)$ with $y(T)=0$. In other words, if the set $R\left(y_{0} ; T\right)$ formed by all reachable states at time $T$ satisfies $R\left(y_{0} ; T\right) \ni 0$.

Of course, the existence of a solution to (1.1) defined in the whole interval $[0, T]$ is not assured for arbitrary $y_{0}$ and $v$, unless something is imposed to $f$. However, for the functions $f$ considered below, at least local existence holds.

During the last years, the null controllability of systems governed by parabolic PDE's has been intensively studied. Nowadays, the situation seems to be the following:

1. In the linear case $(f(s)=a s$ for some $a)$, (1.1) is null controllable with no restriction on $y_{0}, T$ or $\mathcal{O}$. For the proof of this result there exist, at least, two approaches. The first one is due to G. Lebeau

Department of Differential Equations and Numerical Analysis, University of Sevilla, Tarfia s/n, E-41012 Sevilla, Spain. E-mail: cara@numer.us .es.

Received by the journal January 8, 1997. Accepted for publication January 31, 1997.

This work has been partially supported by D.G.I.C.Y.T. (Spain), Proyecto PB95-1242.

(C) Société de Mathématiques Appliquées et Industrielles. Typeset by $\mathrm{IAT}_{\mathrm{E} X} \mathrm{X}$. 
and L. Robbiano. It connects null controllability to the observability through $\mathcal{O} \times(0, T)$ of the solutions to the adjoint system

$$
\left\{\begin{array}{l}
-\partial_{t} \varphi-\Delta \varphi+a \varphi=0 \text { in } Q, \\
\varphi=0 \text { on } \Sigma, \\
\varphi(T)=\varphi^{0}
\end{array}\right.
$$

i.e. to the fact that, for some $C$ only depending on $\Omega, \mathcal{O}$ and $T$, one has

$$
|\varphi(0)|^{2} \leq C \iint_{\mathcal{O} \times(0, T)}|\varphi|^{2} \quad \forall \varphi^{0} \in H
$$

In the particular case $a=0$, a proof of (1.3) is given in [8] (see also $[9])$.

A second approach, due to A. V. Fursikov and O. Yu. Imanuvilov, is based on some global Carleman's inequalities that can be deduced for the solutions to (1.2) (and lead in particular to (1.3)). This method can be applied to quite general linear parabolic problems and provides a control $v$ such that $y(T)=0$ and $y$ is of minimal norm (see [5], [3]). It will be revisited below, in section 5 .

2. When $f$ is sublinear at infinity, i.e.

$$
|f(s)| \leq C|s|+C,
$$

system (1.1) is again null controllable with no restriction on $y_{0}, T$ or $\mathcal{O}$. This is due to O. Yu. Imanuvilov (see [6]). The argument uses a fixed-point reformulation of the problem and the null controllability properties of linear problems. The assumption (1.4) leads to suitable uniform estimates and allows to apply Schauder's theorem.

3. In general, when $f$ is superlinear at infinity, (1.1) is not null controllable. Indeed, recall that when $f(s)=-s \cdot(\log |s|)^{r}(r>1)$ blow-up phenomena may occur. Even when $f$ has "the good sign", null controllability may fail. More precisely, when

$$
C|s|^{r+1}-C \leq f(s) \cdot s \leq C|s|^{r+1}+C \quad(r>1)
$$

and $y_{0} \neq 0$, there exists $T_{0}>0$ such that, if $T<T_{0},(1.1)$ is not null controllable (however, under this assumption on $f$, there exists $T_{1}$ such that, for all $y_{0} \in H,(1.1)$ is null controllable whenever $T>T_{1}$; see [5] and $[6])$.

Hence, the natural question is: What happens if $|f(s)|$ grows faster than $|s|$ but slower than all $|s|^{r}(r>1)$ as $|s| \rightarrow \infty$ ?

We are also interested in questions of this kind for systems controlled on a portion of the boundary. More precisely, let $\gamma$ be a nonempty open subset of $\partial \Omega$ and let us put

$$
\Gamma=\partial \Omega \backslash \gamma
$$

For each $h \in L^{2}(\Sigma)$, we consider the parabolic problem

$$
\left\{\begin{array}{l}
\partial_{t} y-\Delta y+f(y)=0 \text { in } Q \\
y=1_{\gamma} h \text { on } \Sigma \\
y(0)=y_{0}
\end{array}\right.
$$


where $f$ is as before and $1_{\gamma}$ is the characteristic function of $\gamma$. We will say that (1.5) is null controllable if there exists $h$ such that the corresponding initial-boundary problem possesses a solution $y \in C^{0}(0, T ; H)$ satisfying $y(T)=0$.

As for system (1.1), the existence of a global solution to (1.5) is not always assured. Moreover, the sense in which a function $y$ can solve (1.5) has to be specified for general $h \in L^{2}(\Sigma)$. Nevertheless, we will work with appropriate functions $f$ and we will find control functions $h$ which are regular enough to guarantee that (1.5) can be solved (at least) in the usual weak sense.

Again, under condition (1.4), system (1.5) is null controllable (cf. [6]; see also [1] for the case of Neumann boundary controllability). It is also true that, for superlinear nonlinearities, (1.5) is not null controllable in general. Consequently, it is also natural to study the case in which $|f(s)|$ grows superlinearly but slower than all $|s|^{r}$.

\section{THE MAIN RESUlTS AND SOME REMARKS}

In this paper, we prove that systems (1.1) and (1.5) are null controllable when $y_{0} \in C^{0}(\bar{\Omega})$ and the behavior of $f(s)$ as $|s| \rightarrow \infty$ is, at most, almost critical. More precisely, one has:

Theorem 2.1. Assume $f: \mathbb{R} \mapsto \mathbb{R}$ is locally Lipschitz-continuous, $f(0)=0$ and

$$
\lim _{|s| \rightarrow \infty} \frac{f(s)}{s \cdot \log |s|}=0
$$

Also, assume that $y_{0} \in C^{0}(\bar{\Omega})$. Then system (1.1) is null controllable.

THEOREM 2.2. Under the assumptions of theorem 2.1, system (1.5) is null controllable. More precisely, there exists $h$, with

$$
1_{\gamma} h \in L^{2}\left(0, T ; H^{3 / 2}(\partial \Omega)\right) \cap L^{\infty}(\Sigma),
$$

such that the corresponding system (1.5) possesses at least one solution

$$
y \in L^{2}\left(0, T ; H^{1}(\Omega)\right) \cap C^{0}(0, T ; H) \cap L^{\infty}(Q)
$$

satisfying $y(T)=0$.

\section{REMARKS.}

1. Assumption (2.1) can be slightly weakened. Indeed, from the proofs in sections 3 and 4 , we see there exists a positive constant $\varepsilon=\varepsilon(\Omega, \mathcal{O}, T)$ such that theorems 2.1 and 2.2 still hold when

$$
|f(s)| \leq \varepsilon|s| \cdot \log |s| \text { for large }|s| \text {. }
$$

On the other hand, the requirement $y_{0} \in C^{0}(\bar{\Omega})$ is unnecessary in practice. It is sufficient to assume the following: The initial data $y_{0}$ and the function $f$ are such that, when $v=0$, system (1.1) possesses a solution $y \in C^{0}(0, \delta ; H)$ which satisfies $y(\delta) \in C^{0}(\bar{\Omega})$. This happens for all $y_{0} \in H$ and for all functions $f$ as in theorems 2.1 and 2.2, as a consequence of the regularizing effect. 
2. We are dealing with quasi-optimal assumptions: recall again that, for $f(s)= \pm|s|^{r-1} s(r>1)$, systems (1.1) and (1.5) are not null controllable in general. Also, recall that blow-up may occur for $f(s)=-s \cdot(\log |s|)^{r}$.

3. Theorems 2.1 and 2.2 hold for more general problems. Thus, one can replace $-\Delta$ by a general linear elliptic operator $A$, with

$$
A y=-\partial_{i}\left(a_{i j}(x) \partial_{j} y\right)+\partial_{i}\left(b_{i}(x) y\right)+c(x) y .
$$

In this case, the coefficients have to be regular enough and the $a_{i j}$ must satisfy the usual ellipticity condition. The nonlinear term can also be of the form $f(x, t, y)$, with $f: Q \times \mathbb{R} \mapsto \mathbb{R}$ being a Carathéodory function satisfying (for instance):

$$
f(x, t, 0) \equiv 0, \quad s \mapsto f(x, t, s) \quad \text { is locally Lipschitz }
$$

and

$$
\lim _{|s| \rightarrow \infty} \frac{f(x, t, s)}{s \cdot \log |s|}=0 \quad \text { uniformly in }(x, t) \in Q .
$$

Furthermore, a nonvanishing additional term $F=F(x, t)$ can be put in the left in the equations in (1.1) and (1.5). The above results will still be true provided $F$ is sufficiently small near $t=T$. For instance, it will suffice to have

$$
\iint_{Q} F(x, t)^{2} e^{\frac{1}{(T-t)^{2}}}<+\infty .
$$

Let us also mention that, for semilinear parabolic equations of higher order, similar results can be obtained.

4. By adapting the arguments in sections 3 and 4, one is led to a new proof of known local results. These must hold for arbitrary locally Lipschitz functions $f$ satisfying $f(0)=0$. More precisely, if $f$ is given, there exists $\delta=\delta(\Omega, \mathcal{O}, T, f)>0$ such that, for every $y_{0} \in C^{0}(\bar{\Omega})$ with $\left\|y_{0}\right\|_{C^{0}} \leq \delta$, a control $v \in L^{2}(0, T ; H)$ can be found such that the corresponding problem (1.1) possesses a solution $y \in C^{0}(0, T ; H)$ which satisfies $y(T)=0$ (compare with the local results in [1], [4] and [6]).

5. System (1.1) is approximately controllable (in $H$ ) if the set $R\left(y_{0} ; T\right)$ formed by all reachable states at time $T$ is dense in $H$. It has been proved in [2] that, under condition (1.4), system (1.1) is approximately controllable with no restriction on $y_{0}, T$ or $\mathcal{O}$. However, to our knowledge, the approximate controllability of (1.1) under assumption (2.1) is an open question. A similar remark concerning the approximate controllability of system (1.5) can be made.

\section{THE PROOF OF THE BOUNDARY CONTROLLABILITY RESULT}

In this section, we prove theorem 2.2. We will first consider the case in which, for instance, $f$ is $C^{1}$ in the open interval $(-1,1)$ and $y_{0} \in C^{0, \beta}(\bar{\Omega})$ for some $\beta \in(0,1)$. We will use the standard norm in $C^{0, \beta}(\bar{\Omega})$, given as follows:

$$
\|z\|_{\infty}+[z]_{\beta}:=\|z\|_{\infty}+\sup _{\bar{\Omega}} \frac{\left|z(x)-z\left(x^{\prime}\right)\right|}{\left|x-x^{\prime}\right|^{\beta}}
$$

Under these assumptions, we are going to rewrite (1.5) together with the equality $y(T)=0$ as a fixed-point equation in the Banach space $Y=L^{\infty}(Q)$. EsAiM: COCV, APRIL 1997, VOL. 2, PP. 87-103 
Then, using (2.1) and some specific properties of linear parabolic equations, we will see that Schauder's theorem can be applied.

Arguments of this kind have already been used in this and other similar contexts (see [2], [6], [10], ... ). Here, since $f$ is not necessarily globally Lipschitz, one sees that the range of the mapping arising in the fixed-point formulation is not bounded (this is also the case for the questions considered in [11]). Thus, the methods in [1] and [6] cannot be directly applied and some extra work has to be made.

Let us put

$$
g(s)= \begin{cases}\frac{f(s)}{s} & \text { for } s \neq 0 \\ f^{\prime}(0) & \text { at } s=0\end{cases}
$$

Then, in view of assumption (2.1), one has:

$$
\forall \delta>0 \quad \exists C_{\delta} \quad \text { such that }|g(s)| \leq C_{\delta}+\delta|\log | s|| \quad \forall s \in \mathbb{R} .
$$

For each $y \in Y$, we consider the following null controllability problem

$$
\left\{\begin{array}{l}
\partial_{t} u-\Delta u+g(y) u=0 \quad \text { in } \quad Q \\
u=1_{\gamma} h \text { on } \Sigma \\
u(0)=y_{0}, \quad u(T)=0
\end{array}\right.
$$

where the state equation is linear. A solution $u=\Lambda(y)$ to (3.3) can be obtained by adapting the method of A.V. Fursikov and O.Yu. Imanuvilov (cf. [5], [3]). More precisely, for the construction of $\Lambda(y)$, we do the following:

a) We introduce $z=z(\cdot ; y)$, with

$$
\left\{\begin{array}{l}
\partial_{t} z-\Delta z+g(y) z=0 \quad \text { in } \quad Q \\
z=0 \text { on } \Sigma \\
z(0)=y_{0}
\end{array}\right.
$$

b) We fix a function $\theta \in C^{\infty}(0, T)$ such that $\theta(t)=1$ near $t=0, \theta(t)=0$ near $t=T$ and $0 \leq \theta \leq 1$. Then, we set $\Lambda(y)=\theta z(\cdot ; y)+w(\cdot ; y)$, with $w(\cdot ; y)$ being (together with $h$ ) the solution furnished by lemma 3.2 to the following problem (see below):

$$
\left\{\begin{array}{l}
\partial_{t} w-\Delta w+g(y) w=-\theta^{\prime}(t) z(x, t ; y) \text { in } Q \\
w=1_{\gamma} h \text { on } \Sigma \\
w(0)=0, \quad w(T)=0 .
\end{array}\right.
$$

Our aim is to find a fixed-point in $Y$ of the mapping $y \mapsto \Lambda(y)$. Obviously, if we are able to prove that such a fixed-point exists, theorem 2.2 will have been demonstrated (at least when $f$ is $C^{1}$ in $(-1,1)$ and $y_{0} \in C^{0, \beta}(\bar{\Omega})$ ).

First, we will show that the mapping $y \mapsto \Lambda(y)$ is well defined and, also, that it maps a ball of $Y$ into itself. We need the following elementary result: Lemma 3.1. Let $a \in Y$ and $z_{0} \in L^{\infty}(\Omega)$ be given. Then the linear problem

$$
\left\{\begin{array}{l}
\partial_{t} z-\Delta z+a(x, t) z=0 \quad \text { in } Q \\
z=0 \text { on } \quad \mathrm{z} \\
z(0)=z_{0}
\end{array}\right.
$$


possesses exactly one solution $z \in L^{2}\left(0, T ; H_{0}^{1}(\Omega)\right) \cap Y$, with

$$
\|z(t)\|_{\infty} \leq e^{t\|a\|_{Y}}\left\|z_{0}\right\|_{\infty} \quad \forall t \in[0, T] .
$$

We will also use the following result, whose proof is given in section 5:

Lemma 3.2. To each couple $\{a, k\}$, with $a, k \in Y$ such that

$$
k=0 \text { for } 0<t<b \text { and } T-b<t<T,
$$

one can assign a solution $\{w, h\}$ to the null linear controllability problem

$$
\left\{\begin{array}{l}
\partial_{t} w-\Delta w+a(x, t) w=k(x, t) \quad \text { in } Q \\
w=1_{\gamma} h \quad \text { on } \quad \Sigma \\
w(0)=0, \quad w(T)=0
\end{array}\right.
$$

in such a way that

$$
\begin{gathered}
w \in L^{2}\left(0, T ; H^{2}(\Omega)\right) \cap Y, \quad \partial_{t} w \in L^{2}(Q), \\
1_{\gamma} h \in L^{2}\left(0, T ; H^{3 / 2}(\partial \Omega)\right) \cap L^{\infty}(\Sigma)
\end{gathered}
$$

and

$$
\|w\|_{L^{2}\left(0, T ; H^{2}(\Omega)\right)}+\left\|\partial_{t} w\right\|_{L^{2}(Q)}+\|w\|_{Y} \leq e^{C\left(1+\|a\|_{Y}\right)}\|k\|_{Y} .
$$

Here, the constant $C$ can depend on $\Omega, \gamma, T$ and $b$, but not on $k$.

REMARK. Lemma 3.2 will also be used in section 4, for the proof of theorem 2.1. It remains true under more general conditions on $k$.

From lemmas 3.1 and 3.2, it is clear that $y \mapsto \Lambda(y)$ is a well defined mapping from $Y$ into itself. Moreover, one has

$$
\|\Lambda(y)\|_{Y} \leq e^{C\left(1+\|g(y)\|_{Y}\right)}\left\|y_{0}\right\|_{\infty} \quad \forall y \in Y,
$$

where $C$ can only depend on $\Omega, \gamma$ and $T$. Hence, in view of (3.2), one sees that $\Lambda$ maps a ball $B_{R}$ of $Y$ into itself.

It is clear that $y \mapsto \Lambda(y)$ is a continuous mapping. Let us now prove that, for some $\alpha \in(0,1)$, it maps bounded sets in $Y$ into bounded sets in $C^{0, \alpha, \alpha / 2}(\bar{Q})$. This will suffice to our purpose, since this space is compactly embedded in $Y$. Recall that $C^{0, \alpha, \alpha / 2}(\bar{Q})$ is the Banach space formed by all functions $u \in C^{0}(\bar{Q})$ such that

$$
[u]_{\alpha, \alpha / 2}:=\sup _{\bar{Q}} \frac{\left|u(x, t)-u\left(x^{\prime}, t\right)\right|}{\left|x-x^{\prime}\right|^{\alpha}}+\sup _{\bar{Q}} \frac{\left|u(x, t)-u\left(x, t^{\prime}\right)\right|}{\left|t-t^{\prime}\right|^{\alpha / 2}}<+\infty .
$$

The (natural) norm in $C^{0, \alpha, \alpha / 2}(\bar{Q})$ is as follows:

$$
\|u\|_{\alpha, \alpha / 2}:=\|u\|_{Y}+[u]_{\alpha, \alpha / 2}
$$

We will use the following lemma, which is proved in [7] (see theorems 7.1 and 10.1, Ch. III):

ESAIM: COCV, APRIL 1997, VOL. 2, PP. 87-103 
Lemma 3.3. Assume $a, k \in Y, \widetilde{h} \in L^{2}\left(0, T ; H^{1 / 2}(\partial \Omega)\right) \cap L^{\infty}(\Sigma)$ and $z_{0} \in$ $C^{0, \beta}(\bar{\Omega})$, where $\beta \in(0,1)$. Then the linear problem

$$
\left\{\begin{array}{l}
\partial_{t} u-\Delta u+a(x, t) u=k(x, t) \quad \text { in } \quad Q \\
u=\widetilde{h} \text { on } \Sigma \\
u(0)=z_{0}
\end{array}\right.
$$

possesses exactly one solution $u \in L^{2}\left(0, T ; H^{1}(\Omega)\right) \cap Y$. Furthermore, there exists $\alpha \in(0, \beta)$ (only depending on $\Omega, T$ and $\beta$ ) such that $u \in C^{0, \alpha, \alpha / 2}(\bar{Q})$ and there exists a function $M_{1}$ (increasing with respect to its last argument) such that

$$
[u]_{\alpha, \alpha / 2} \leq M_{1}\left(\Omega, T, \beta, \alpha,\left\|z_{0}\right\|_{\infty}+\left[z_{0}\right]_{\beta},\|u\|_{Y}\right) .
$$

We deduce that, for some $\alpha$, all functions $\Lambda(y)$ belong to $C^{0, \alpha, \alpha / 2}(\bar{Q})$. Combining (3.12) with (3.11), we also deduce that, for some $M_{2}$ (again increasing with respect to its last argument), one has:

$$
\|\Lambda(y)\|_{\alpha, \alpha / 2} \leq M_{2}\left(\Omega, T, \beta, \alpha,\left\|y_{0}\right\|_{\infty}+\left[y_{0}\right]_{\beta},\|g(y)\|_{Y}\right) .
$$

This shows that $\Lambda$ maps bounded sets of $Y$ into bounded sets of $C^{0, \alpha, \alpha / 2}(\bar{Q})$.

We have thus seen that Schauder's theorem can be applied to the fixedpoint equation $y=\Lambda(y), y \in Y$. This proves theorem 2.2 when $f$ is $C^{1}$ in $(-1,1)$ and $y_{0} \in C^{0, \beta}(\bar{\Omega})$.

In the general case, one can always put

$$
f=\lim _{n \rightarrow \infty} f_{n} \quad \text { uniformly in } \mathbb{R},
$$

for some locally Lipschitz functions $f_{n}$ which are $C^{1}$ in $(-1,1)$ and satisfy $f_{n}(0)=0$, and $f_{n}=f$ outside $(-2,2)$. One can also put

$$
y_{0}=\lim _{n \rightarrow \infty} y_{0}^{n} \quad \text { uniformly in } \bar{\Omega} \text {, }
$$

for some functions $y_{0}^{n} \in C^{0, \beta}(\bar{\Omega})$. For each $n \geq 1$, let $\left\{y_{n}, h_{n}\right\}$ be a couple satisfying

$$
\left\{\begin{array}{l}
\partial_{t} y_{n}-\Delta y_{n}+f_{n}\left(y_{n}\right)=0 \quad \text { in } \quad Q \\
y_{n}=1_{\gamma} h_{n} \text { on } \Sigma \\
y_{n}(0)=y_{0}^{n}, \quad y_{n}(T)=0
\end{array}\right.
$$

constructed as above. For

$$
\begin{aligned}
& g_{n}(s)=\frac{f_{n}(s)}{s} \text { for } s \neq 0, \\
& f_{n}^{\prime}(0) \quad \text { at } s=0,
\end{aligned}
$$

it is not difficult to see using (2.1) that

$\forall \delta>0 \quad \exists C_{\delta} \quad$ such that $\left|g_{n}(s)\right| \leq C_{\delta}+\delta|\log | s|| \quad \forall s \in \mathbb{R} \forall n \geq 1$.

Consequently, one has $\left\|y_{n}\right\|_{Y} \leq$ Const. (use the corresponding estimates (3.11)). Notice that $y_{n}$ can be written in the form $y_{n}=\theta z_{n}+w_{n}$, where

- $z_{n}$ is uniformly bounded in $L^{2}\left(0, T ; H_{0}^{1}(\Omega)\right) \cap Y$ and $\partial_{t} z_{n}$ is uniformly bounded in $L^{2}\left(0, T ; H^{-1}(\Omega)\right)$, see $(3.5)$ and (3.6).

- $w_{n}$ can be estimated as in (3.10). 
Hence, it can be assumed that $y_{n}$ converges strongly in $L^{2}(Q)$ and a.e. and $1_{\gamma} h_{n}$ converges (at least) weakly in $L^{2}\left(0, T ; H^{3 / 2}(\partial \Omega)\right)$. We can thus take limits in (3.13) as $n \rightarrow \infty$ and obtain (1.5) together with the equality $y(T)=$ 0 . This proves theorem 2.2 .

\section{THE PROOF OF THE INTERNAL CONTROLLABILITY RESUlT}

In this section, we will prove theorem 2.1. As in the previous section, we will first assume that $f$ is $C^{1}$ in the open interval $(-1,1)$ and $y_{0} \in C^{0, \beta}(\bar{\Omega})$ for some $\beta \in(0,1)$. Again, we will use the function $g$ given by (3.1).

We will introduce a mapping $y \mapsto \Pi(y)$ such that, for each $y \in Y, u=$ $\Pi(y)$ is, together with some $v \in L^{2}(0, T ; H)$, a solution to the following problem:

$$
\left\{\begin{array}{l}
\partial_{t} u-\Delta u+g(y) u=1_{\mathcal{O}} v \quad \text { in } \quad Q \\
u=0 \text { on } \quad \Sigma \\
u(0)=y_{0}, \quad u(T)=0
\end{array}\right.
$$

This is made as follows:

a) First, we fix a nonempty open, simply connected and regular set $\mathcal{O}^{\prime} \subset \subset$ $\mathcal{O}$, we put

$$
\gamma=\partial \mathcal{O}^{\prime}, \quad \Gamma=\partial \Omega
$$

and we denote by $D$ the open set bounded by $\gamma$ and $\Gamma$, i.e. $D=\Omega \backslash \overline{\mathcal{O}^{\prime}}$.

b) Using lemma 3.2 in $D \times(0, T)$, we assign to $y$ a couple $\{\hat{w}(\cdot ; y), \hat{h}(\cdot ; y)\}$ satisfying

$$
\left\{\begin{array}{l}
\partial_{t} \hat{w}-\Delta \hat{w}+g(y) \hat{w}=-\theta^{\prime}(t) z(x, t ; y) \text { in } D \times(0, T) \\
\hat{w}=1_{\gamma} \hat{h} \text { on } \partial D \times(0, T), \\
\hat{w}(0)=0 \text { and } \hat{w}(T)=0 \text { in } D .
\end{array}\right.
$$

Here, $z=z(\cdot ; y)$ is the (unique) solution to (3.4). One has

$$
\begin{gathered}
\hat{w} \in L^{2}\left(0, T ; H^{2}(D)\right) \cap L^{\infty}(D \times(0, T)), \quad \partial_{t} \hat{w} \in L^{2}(D \times(0, T)), \\
1_{\gamma} \hat{h} \in L^{2}\left(0, T ; H^{3 / 2}(\partial D)\right) \cap L^{\infty}(\partial D \times(0, T))
\end{gathered}
$$

and

$$
\|\hat{w}\|_{L^{\infty}(D \times(0, T))} \leq e^{C\left(1+\|g(y)\|_{Y}\right)}\left\|y_{0}\right\|_{\infty}
$$

where $C$ can depend only on $\Omega, \mathcal{O}^{\prime}$ and $T$. We will denote by $\bar{w}$ the extensionby-zero of $\hat{w}$ to the whole set $Q$.

c) Let us set $w=\eta \bar{w}$ and $v=-2 \nabla \eta \cdot \nabla \bar{w}-(\Delta \eta) \bar{w}+(1-\eta) \theta^{\prime} z(\cdot ; y)$. Here, $\eta=\eta(x)$ is a $C^{\infty}$ cut-off function satisfying:

$$
\begin{array}{ll}
\eta=1 & \text { in a neighborhood of } \Omega \backslash \mathcal{O}, \\
\eta=0 & \text { in a neighborhood of } \mathcal{O}^{\prime}
\end{array}
$$

and $0 \leq \eta \leq 1$. Then, we put $\Pi(y)=\theta z(\cdot ; y)+w(\cdot ; y)$, with $\theta$ being as in section 3, i.e. a function satisfying $\theta \in C^{\infty}(0, T), \theta(t)=1$ near $t=0$, $\theta(t)=0$ near $t=T$ and $0 \leq \theta \leq 1$.

EsAiM: COCV, APRIL 1997, VOL. 2, PP. 87-103 
It is not difficult to see that $u=\Pi(y)$ is (together with $v$ ) a solution to (4.1). From (4.3)-(4.5) and (3.6), one has

$$
u \in L^{2}\left(0, T ; H_{0}^{1}(\Omega)\right) \cap Y, \quad v \in L^{2}(0, T ; H)
$$

and

$$
\|\Pi(y)\|_{Y} \leq e^{C\left(1+\|g(y)\|_{Y}\right)}\left\|y_{0}\right\|_{\infty}
$$

Consequently, $\Pi$ maps a ball $B_{R}$ of $Y$ into itself. It is also clear that $y \mapsto$ $\Pi(y)$ is a continuous mapping. Furthermore, we can apply lemma 3.3 to (4.2). This leads easily to the facts that $\Pi(y) \in C^{0, \alpha, \alpha / 2}(\bar{Q})$ and

$$
\|\Pi(y)\|_{\alpha, \alpha / 2} \leq M_{3}\left(\Omega, T, \beta, \alpha,\left\|y_{0}\right\|_{\infty}+\left[y_{0}\right]_{\beta},\|g(y)\|_{Y}\right)
$$

for all $y \in Y$ and for some $\alpha \in(0, \beta)$ (once more, $M_{3}$ is increasing with respect to its last argument). Hence, $\Pi$ maps bounded sets of $Y$ into bounded sets of $C^{0, \alpha, \alpha / 2}(\bar{Q})$ and we can apply Schauder's theorem to the fixed-point equation $y=\Pi(y), y \in Y$. This proves theorem 2.1 when $f$ is $C^{1}$ in $(-1,1)$ and $y_{0} \in C^{0, \beta}(\bar{\Omega})$.

In order to prove the same result in the general case, we can argue as in section 3. Thus, introducing the functions $f_{n}$ and $y_{0}^{n}$, we find for each $n \geq 1$ a couple $\left\{y_{n}, v_{n}\right\}$ satisfying

$$
\left\{\begin{array}{l}
\partial_{t} y_{n}-\Delta y_{n}+f_{n}\left(y_{n}\right)=1_{\mathcal{O}} v_{n} \text { in } Q \\
y_{n}=0 \text { on } \Sigma \\
y_{n}(0)=y_{0}^{n}, \quad y_{n}(T)=0
\end{array}\right.
$$

and

$$
\left\|y_{n}\right\|_{Y} \leq \text { Const., } \quad\left\|v_{n}\right\|_{L^{2}(0, T ; H)} \leq \text { Const. }
$$

Hence, it can be assumed that $y_{n}$ converges strongly in $L^{2}(Q)$ and a.e. and $1_{\gamma} v_{n}$ converges (at least) weakly in $L^{2}(Q)$. Taking limits in (4.6), we obtain (1.1) and $y(T)=0$. This proves theorem 2.1.

\section{The PRoOf of LEMMA 3.2}

We will first analyze a null controllability problem, similar to (3.7), in a suitable larger domain $G \times(0, T)$. We will present an argument, taken from [5] and [3], which leads to a function $\widetilde{w}$ that solves this problem in a generalized sense. Then, we will assign to $\widetilde{w}$ a couple $\{w, h\}$ satisfying (3.7) and we will check that (3.8), (3.9) and (3.10) are satisfied. Accordingly, the proof will be divided in several steps.

Step 1: The Analysis of an AUXiliary PRoblem - Let $\Omega$ and $\gamma \subset \partial \Omega$ be as before and assume that $a, k \in Y$, with $k=0$ for $0<t<b$ and also for $T-b<t<T$. Let $G \subset \mathbb{R}^{N}$ be a bounded, regular and open set with $G \supset \Omega$ and $\partial \Omega \cap \partial G=\Gamma=\partial \Omega \backslash \gamma$. Let $S_{0} \subset \partial G$ be an open neighborhood of $\Gamma\left(S_{0} \neq \partial G\right)$ and let us put

$$
S_{1}=\partial G \backslash S_{0}, \quad \tilde{\Sigma}=\partial G \times(0, T) \text { and } \quad \tilde{\Sigma}_{i}=S_{i} \times(0, T)
$$

for $i=1,2$. We will denote by $\widetilde{a}$ and $\widetilde{k}$ the extensions-by-zero to the whole set $G \times(0, T)$ of the functions $a$ and $k$, respectively. We will need the following 
LEMMA 5.1. There exists a positive function $\rho^{0} \in C^{2}(\bar{G})$ such that $\nabla \rho^{0} \neq 0$ in $\bar{G}$ and $\partial_{n} \rho^{0} \leq 0$ on $S_{0}$.

Here (and also in the sequel), $\partial_{n}$ stands for the outward normal derivative. Lemma 5.1 is proved in [6] (see [1] for another similar but different result). Let us introduce the space

$$
Z_{0}=\left\{q \in C^{2}(\bar{G} \times[0, T]) ; q=0 \text { on } \widetilde{\Sigma}, \partial_{n} q=0 \text { on } \widetilde{\Sigma}_{1}\right\} .
$$

We will need a second lemma:

LEMMA 5.2. There exists a positive function $\rho$ (which is of class $C^{2}$ in the set $\bar{G} \times(0, T)$ and depends only on $G, S_{1}$ and $\left.T\right)$ and there exist constants $s_{*}\left(G, S_{1}, T,\|a\|_{Y}\right)$ and $C_{*}\left(G, S_{1}, T\right)$ such that the following inequalities hold for all $s \geq s_{*}$ and for all $q \in Z_{0}$ :

$$
\left\{\begin{array}{l}
\frac{1}{s} \iint \rho^{-2 s} t(T-t)\left(\left|\partial_{t} q\right|^{2}+|\Delta q|^{2}\right) \\
\quad+s \iint \rho^{-2 s} t^{-1}(T-t)^{-1}|\nabla q|^{2}+s^{3} \iint \rho^{-2 s} t^{-3}(T-t)^{-3}|q|^{2} \\
\quad \leq C_{*} \iint \rho^{-2 s}\left|\partial_{t} q+\Delta q-\widetilde{a} q\right|^{2} .
\end{array}\right.
$$

Here, the integrals are extended to $G \times(0, T)$. Furthermore, $s_{*}$ can be chosen of the form $\sigma_{1}+\sigma_{2}\|a\|_{Y}^{2 / 3}$, where $\sigma_{1}$ and $\sigma_{2}$ only depend on $G, S_{1}$ and $T$.

The inequalities (5.1) are global Carleman's estimates associated to the adjoint system (1.2). The main ideas needed for the proof of lemma 5.2 are presented in [5] and [3]. Nevertheless, in order to clarify the situation as much as possible, we will give a complete proof of this lemma in the following section. This will serve to show that we can choose $s_{*}$ as indicated above. Also, it will be seen that an admissible choice for $\rho$ is

$$
\rho(x, t)=\exp \left(\frac{\rho^{1}(x)}{t(T-t)}\right)
$$

where $\rho^{1}=\exp (\lambda \bar{\rho})-\exp \left(\lambda \rho^{0}\right), \lambda$ and $\bar{\rho}$ are large enough and $\rho^{0}$ is the function furnished by lemma 5.1 .

Let us come back to the proof of lemma 3.2. Let us fix $s$ and $\rho$ such that (5.1) holds. Then

$$
[p, q]=\iint_{G \times(0, T)} \rho^{-2 s}\left(\partial_{t} p+\Delta p-\widetilde{a} p\right)\left(\partial_{t} q+\Delta q-\widetilde{a} q\right)
$$

is a scalar product in $Z_{0}$. Let $Z$ be the completion of $Z_{0}$ for $[\cdot, \cdot \cdot$. If $q \in Z$, the functions $\rho^{-2 s} t(T-t)\left|\partial_{t} q\right|^{2}, \rho^{-2 s} t(T-t)|\Delta q|^{2}$, etc. are integrable and (5.1) is satisfied. Let us put

$$
\langle l, q\rangle=-\iint_{G \times(0, T)} \tilde{k} q \quad \forall q \in Z .
$$

Then, $l$ is a bounded linear form on $Z$. Indeed, using Hölder's inequality and (5.1), one has:

$$
\left|\iint_{G \times(0, T)} \tilde{k} q\right| \leq E\left(G, S_{1}, T, k, s\right)[q, q]^{1 / 2} \quad \forall q \in Z,
$$


with

$$
E\left(G, S_{1}, T, k, s\right)=C_{*}\left(G, S_{1}, T\right)^{1 / 2} s^{-3 / 2}\left(\iint_{Q} \rho^{2 s} t^{3}(T-t)^{3}|k|^{2}\right)^{1 / 2} .
$$

But this is a finite quantity, in view of the assumptions on $k$. Consequently, there exists a unique solution $p$ to the following problem:

$$
[p, q]=\langle l, q\rangle \quad \forall q \in Z, \quad p \in Z .
$$

Let us put $\widetilde{w}=\rho^{-2 s}\left(\partial_{t} p+\Delta p-\widetilde{a} p\right)$. Obviously, $\widetilde{w} \in L^{2}(G \times(0, T))$ (in fact, one has more than this); moreover, from lemma 5.2, (5.2) and (5.3), it is easy to deduce that

$$
\|\widetilde{w}\|_{L^{2}(G \times(0, T))} \leq e^{C\left(1+\|a\|_{Y}^{2 / 3}\right)}\|k\|_{L^{2}(Q)},
$$

where $C$ only depends on $G, S_{1}, T$ and $b$. In the next step, it will be shown that $\widetilde{w}$ is a generalized solution to a null controllability problem (in $G \times(0, T))$.

Step 2: Properties of $\widetilde{w}-$ In the sequel, new constants $C$ will appear, all them depending only on $G, S_{1}, T$ and $b$. One has $\partial_{t} \widetilde{w}-\Delta \widetilde{w}+\widetilde{a} \widetilde{w}=\widetilde{k}$ in the usual distributional sense in $G \times(0, T)$ since, for all $\Phi \in \mathcal{D}(G \times(0, T))$, one has

$$
\left\langle\partial_{t} \widetilde{w}-\Delta \widetilde{w}+\widetilde{a} \widetilde{w}, \Phi\right\rangle=-\iint_{G \times(0, T)} \widetilde{w}\left(\partial_{t} \Phi+\Delta \Phi-\widetilde{a} \Phi\right)=\iint_{G \times(0, T)} \widetilde{k} \Phi .
$$

One also has $\widetilde{w} \in C^{0}\left(0, T ; H^{-1}(G)\right)$ and $\widetilde{w}(0)=\widetilde{w}(T)=0$. This stems from the following two facts:

- $\widetilde{w} \in L^{2}(G \times(0, T))$ and $\partial_{t} \widetilde{w} \in L^{2}\left(0, T ; H^{-2}(G)\right)$ (because $\partial_{t} \widetilde{w}-\Delta \widetilde{w}+$ $\left.\widetilde{a} \widetilde{w}=\widetilde{k} \in L^{\infty}(G \times(0, T))\right)$. Consequently, $\widetilde{w} \in C^{0}\left(0, T ; H^{-1}(G)\right)$.

- If $\Phi_{1} \in \mathcal{D}(G), \Phi_{2} \in C^{1}[0, T]$ and $\Phi=\Phi_{1}(x) \Phi_{2}(t)$, one has at the same time

$$
\begin{aligned}
& \iint_{G \times(0, T)} \tilde{k} \Phi=\iint_{G \times(0, T)}\left(\partial_{t} \widetilde{w}-\Delta \widetilde{w}+\widetilde{a} \widetilde{w}\right) \Phi \\
& =-\iint_{G \times(0, T)} \widetilde{w}\left(\partial_{t} \Phi+\Delta \Phi-\widetilde{a} \Phi\right)+\left.\langle\widetilde{w}, \Phi\rangle\right|_{t=0} ^{t=T}
\end{aligned}
$$

(as a consequence of the previous regularity of $\widetilde{w}$ and $\partial_{t} \widetilde{w}$ ) and also

$$
\iint_{G \times(0, T)} \widetilde{k} \Phi=-\iint_{G \times(0, T)} \widetilde{w}\left(\partial_{t} \Phi+\Delta \Phi-\widetilde{a} \Phi\right)
$$

(from the definition of $\widetilde{w}$ ). Hence, $\widetilde{w}(0)=\widetilde{w}(T)=0$ in $H^{-1}(G)$ (it was reasonable to expect something like this, since $\widetilde{w}$ is the product of $\rho^{-s}$ by a function of $\left.L^{2}(G \times(0, T))\right)$.

Furthermore, $\widetilde{w}=0$ on $\widetilde{\Sigma}_{0}=S_{0} \times(0, T)$ in an appropriate sense. Indeed, one has:

- $\widetilde{w} \in L^{2}(G \times(0, T))$ and $\Delta \widetilde{w} \in H^{-1}\left(0, T ; L^{2}(G)\right)$, whence $\widetilde{w}$ possesses a trace in $H^{-1}\left(0, T ; H^{-1 / 2}(\partial G)\right)$. 
- If $\Phi_{1} \in C^{2}(\bar{G}), \Phi_{2} \in \mathcal{D}(0, T)$ and $\Phi=\Phi_{1}(x) \Phi_{2}(t)$ belongs to $Z_{0}$, one must have (5.5) and also

$$
\begin{aligned}
& \iint_{G \times(0, T)} \widetilde{k} \Phi=\iint_{G \times(0, T)}\left(\partial_{t} \widetilde{w}-\Delta \widetilde{w}+\widetilde{a} \widetilde{w}\right) \Phi \\
& =-\iint_{G \times(0, T)} \widetilde{w}\left(\partial_{t} \Phi+\Delta \Phi-\widetilde{a} \Phi\right)+\left\{\widetilde{w}, \partial_{n} \Phi\right\},
\end{aligned}
$$

as a consequence of the previous property. Here, $\{\cdot, \cdot\}$ stands for the duality pairing concerning the spaces $H^{-1}\left(0, T ; H^{-1 / 2}(\partial G)\right)$ and $H_{0}^{1}\left(0, T ; H^{1 / 2}(\partial G)\right)$. Thus, the trace of $\widetilde{w}$ on $\widetilde{\Sigma}_{0}$ vanishes, as an element of $H^{-1}\left(0, T ; H^{-1 / 2}\left(S_{0}\right)\right)$.

From the previous considerations, one sees that $\widetilde{w}$ solves, in some generalized sense, the following problem:

$$
\left\{\begin{array}{l}
\partial_{t} \widetilde{w}-\Delta \widetilde{w}+\widetilde{a}(x, t) \widetilde{w}=\widetilde{k}(x, t) \quad \text { in } \quad G \times(0, T), \\
\widetilde{w}=0 \text { on } \widetilde{\Sigma}_{0}, \\
\widetilde{w}(0)=0 \text { and } \widetilde{w}(T)=0 \text { in } \quad G .
\end{array}\right.
$$

Step 3: The Definition and properties of $w$ and $h$ - Let us denote by $w$ the restriction to $Q$ of $\widetilde{w}$. Let $\eta_{0} \in C^{\infty}(\bar{G})$ be a cut-off function with supp $\eta_{0} \subset G \cup S_{0}, \eta_{0} \equiv 1$ in a neighborhood of $\Omega$ and $0 \leq \eta_{0} \leq 1$. Let us set $\widetilde{w}_{0}=\eta_{0} \widetilde{w}$. A simple computation shows that $\widetilde{w}_{0}$ satisfies

$$
\left\{\begin{array}{l}
\partial_{t} \widetilde{w}_{0}-\Delta \widetilde{w}_{0}+\widetilde{a}(x, t) \widetilde{w}_{0}=\widetilde{g}_{0}(x, t) \text { in } G \times(0, T), \\
\widetilde{w}_{0}=0 \text { on } \widetilde{\Sigma}, \\
\widetilde{w}_{0}(0)=0 \text { and } \widetilde{w}_{0}(T)=0 \text { in } G,
\end{array}\right.
$$

where $\widetilde{g}_{0}=\eta_{0} \widetilde{k}-2 \nabla \eta_{0} \cdot \nabla \widetilde{w}-\left(\Delta \eta_{0}\right) \widetilde{w}$, is a function in $L^{2}\left(0, T ; H^{-1}(G)\right)$. Hence, $\widetilde{w}_{0} \in L^{2}\left(0, T ; H_{0}^{1}(G)\right) \cap C^{0}\left(0, T ; L^{2}(G)\right)$ and $w \in L^{2}\left(0, T ; H^{1}(\Omega)\right) \cap$ $C^{0}(0, T ; H)$. It follows that $w$ satisfies $(3.7)$ for some $h$. From the usual energy estimates, the definition of $\widetilde{g}_{0}$ and (5.4), one also has:

$$
\left\|\widetilde{w}_{0}\right\|_{L^{2}\left(0, T ; H_{0}^{1}(G)\right)}+\left\|\widetilde{w}_{0}\right\|_{L^{\infty}\left(0, T ; L^{2}(G)\right)} \leq e^{C\left(1+\|a\|_{Y}\right)}\|k\|_{L^{2}(Q)} .
$$

It remains to see that $(3.8),(3.9)$ and $(3.10)$ hold.

Let $\eta_{1} \in C^{\infty}(\bar{G})$ be a second cut-off function such that $\eta_{1} \equiv 1$ in a neighborhood of $\Omega, 0 \leq \eta_{1} \leq 1$ and supp $\eta_{1} \subset\left\{\eta_{0}=1\right\}$. Let us introduce the new function $\widetilde{w}_{1}=\eta_{1} \widetilde{w}$ (which coincides with $\eta_{1} \widetilde{w}_{0}$ ). Then

$$
\left\{\begin{array}{l}
\partial_{t} \widetilde{w}_{1}-\Delta \widetilde{w}_{1}+\widetilde{a}(x, t) \widetilde{w}_{1}=\widetilde{g}_{1}(x, t) \text { in } G \times(0, T), \\
\widetilde{w}_{1}=0 \text { on } \tilde{\Sigma}, \\
\widetilde{w}_{1}(0)=0 \text { and } \widetilde{w}_{1}(T)=0 \text { in } G,
\end{array}\right.
$$

where $\widetilde{g}_{1}=\eta_{1} \widetilde{k}-2 \nabla \eta_{1} \cdot \nabla \widetilde{w}_{0}-\left(\Delta \eta_{1}\right) \widetilde{w}_{0}$, is a function in $L^{2}(G \times(0, T))$. Hence, $\widetilde{w}_{1} \in L^{2}\left(0, T ; H^{2}(G)\right) \cap C^{0}\left(0, T ; H_{0}^{1}(G)\right), \partial_{t} \widetilde{w}_{1} \in L^{2}(G \times(0, T))$; once more, from the energy estimates, the definition of $\widetilde{g}_{1}$ and (5.6), one finds:

$$
\left\{\begin{array}{c}
\left\|\widetilde{w}_{1}\right\|_{L^{2}\left(0, T ; H^{2}(G)\right)}+\left\|\widetilde{w}_{1}\right\|_{L^{\infty}\left(0, T ; H_{0}^{1}(G)\right)}+\left\|\partial_{t} \widetilde{w}_{1}\right\|_{L^{2}(G \times(0, T))} \\
\leq e^{C\left(1+\|a\|_{Y}\right)}\left\|\widetilde{g}_{1}\right\|_{L^{2}(G \times(0, T))} \leq e^{C\left(1+\|a\|_{Y}\right)}\|k\|_{Y} .
\end{array}\right.
$$


Since $w$ is the restriction to $Q$ of the function $\widetilde{w}_{1}$, we have actually proved (3.8), (3.9) and (3.10), except for the estimate of $\|w\|_{Y}$.

Now, we can introduce a third cut-off function $\eta_{2} \in C^{\infty}(\bar{G})$ such that, $\eta_{2} \equiv 1$ in a neighborhood of $\Omega, 0 \leq \eta_{2} \leq 1$ and supp $\eta_{2} \subset\left\{\eta_{1}=1\right\}$. This leads to $\widetilde{w}_{2}=\eta_{2} \widetilde{w}$ (which coincides with $\eta_{2} \widetilde{w}_{1}$ ) and we see that

$$
\left\{\begin{array}{l}
\partial_{t} \widetilde{w}_{2}-\Delta \widetilde{w}_{2}+\widetilde{a}(x, t) \widetilde{w}_{2}=\widetilde{g}_{2}(x, t) \quad \text { in } G \times(0, T), \\
\widetilde{w}_{2}=0 \text { on } \tilde{\Sigma}, \\
\widetilde{w}_{2}(0)=0 \text { and } \widetilde{w}_{2}(T)=0 \text { in } G
\end{array}\right.
$$

where $\widetilde{g}_{2} \in L^{2}\left(0, T ; H^{1}(G)\right) \cap L^{\infty}\left(0, T ; L^{2}(G)\right)$.

For simplicity, let us assume that $N \leq 3$ (a similar argument holds when $N \geq 4$ after introducing additional cut-off functions). Then, $\widetilde{g}_{2} \in$ $L^{2}\left(0, T ; L^{\overline{6}}(G)\right)$. Consequently, we can use theorem 6.1 (Ch. II) and theorem 7.1 (Ch. III) of [7], which give $\widetilde{w}_{2} \in L^{\infty}(G \times(0, T))$ and

$$
\left\|\widetilde{w}_{2}\right\|_{L^{\infty}(G \times(0, T))} \leq C\left(\|a\|_{Y}+\left\|\widetilde{g}_{2}\right\|_{L^{2}\left(0, T ; L^{6}(G)\right)}\right)^{p}
$$

for some $p>0$. This yields the desired estimate for $\|w\|_{Y}$. Hence, lemma 3.2 is proved.

\section{The PRoof of lemma 5.2}

In this section, $\|\cdot\|$ and $((\cdot, \cdot))$ will stand for the norm and the scalar product in $L^{2}(G \times(0, T))$, respectively. We will use the standard convention for the sum of repeated indices. Furthermore, all integrals below are extended to $G \times(0, T)$ (unless otherwise specified).

We will denote by $A$ and $C$ generic constants which can depend only on the data $G, S_{1}$ and $T$. In our argument, other (specific) constants $\lambda_{1}, s_{1}$, etc. depending on the same data will also be needed. Let us put

$$
\varphi(x, t)=\frac{\rho^{1}(x)}{t(T-t)} \quad \text { and } \quad \rho(x, t)=\exp \left(\frac{\rho^{1}(x)}{t(T-t)}\right),
$$

where $\rho^{1}=\exp (\lambda \bar{\rho})-\exp \left(\lambda \rho^{0}\right), \lambda$ is large enough (its choice is made below), $\bar{\rho}>\rho^{0}$ in $\bar{G}$ and $\rho^{0}$ is the function given by lemma 5.1. We will prove (5.1) for this function $\rho$.

Let $q$ be given in $Z_{0}$ and set $\psi=\rho^{-s} q$, where $s$ is also chosen below. Let us put $w=\partial_{t} q+\Delta q-\widetilde{a} q$. Then

$$
\begin{aligned}
& M_{1} \psi+M_{2} \psi \\
& \quad=\left(\partial_{t} \psi+2 s \nabla \varphi \cdot \nabla \psi\right)+\left(\Delta \psi+s^{2}|\nabla \varphi|^{2} \psi+s\left(\partial_{t} \varphi\right) \psi\right) \\
& \quad=w_{s}-(s(\Delta \varphi)-\widetilde{a}) \psi
\end{aligned}
$$

where $w_{s}=\rho^{-s} w$. We deduce the following identity:

$$
\left\|M_{1} \psi\right\|^{2}+\left\|M_{2} \psi\right\|^{2}+2\left(\left(M_{1} \psi, M_{2} \psi\right)\right)=\left\|w_{s}-(s(\Delta \varphi)-\widetilde{a}) \psi\right\|^{2}
$$


Now, let us analyze the scalar product in $(6.2)$ :

$$
\begin{aligned}
& \left(\left(M_{1} \psi, M_{2} \psi\right)\right) \\
& \quad=\iint \partial_{t} \psi \Delta \psi+2 s \iint(\nabla \varphi \cdot \nabla \psi) \Delta \psi \\
& \quad+s^{2} \iint \partial_{t} \psi|\nabla \varphi|^{2} \psi+2 s^{3} \iint(\nabla \varphi \cdot \nabla \psi)|\nabla \varphi|^{2} \psi \\
& \quad+s \iint \partial_{t} \psi\left(\partial_{t} \varphi\right) \psi+2 s^{2} \iint(\nabla \varphi \cdot \nabla \psi)\left(\partial_{t} \varphi\right) \psi \\
& \quad=I_{1}+2 s I_{2}+s^{2} I_{3}+2 s^{3} I_{4}+s I_{5}+2 s^{2} I_{6} .
\end{aligned}
$$

First, one has:

$$
I_{1}=-\frac{1}{2} \iint \partial_{t}|\nabla \psi|^{2}=0 .
$$

After integration by parts and some manipulation, one easily finds:

$$
I_{2}=\iint\left(-\partial_{i} \partial_{j} \varphi+\frac{1}{2}(\Delta \varphi) \delta_{i j}\right) \partial_{i} \psi \partial_{j} \psi+\frac{1}{2} \iint_{\widetilde{\Sigma}_{0}} \partial_{n} \varphi\left|\partial_{n} \psi\right|^{2} .
$$

Also,

$$
I_{3}=-\frac{1}{2} \iint \partial_{t}|\nabla \varphi|^{2}|\psi|^{2}, \quad I_{5}=-\frac{1}{2} \iint \partial_{t}^{2} \varphi|\psi|^{2}
$$

and

$$
I_{6}=-\frac{1}{2} \iint\left(\partial_{i}\left(\partial_{t} \varphi\right) \partial_{i} \varphi+\partial_{t} \varphi \Delta \varphi\right)|\psi|^{2} .
$$

Finally, integration by parts also leads to

$$
I_{4}=\iint\left(-\partial_{i} \partial_{j} \varphi-\frac{1}{2}(\Delta \varphi) \delta_{i j}\right) \partial_{i} \varphi \partial_{j} \varphi|\psi|^{2}
$$

Consequently,

$$
\begin{aligned}
& \left\|M_{1} \psi\right\|^{2}+\left\|M_{2} \psi\right\|^{2}+4 s^{3} \iint\left(-\partial_{i} \partial_{j} \varphi\right) \partial_{i} \varphi \partial_{j} \varphi|\psi|^{2} \\
& \quad+2 s \iint_{\widetilde{\Sigma}_{0}} \partial_{n} \varphi\left|\partial_{n} \psi\right|^{2}+2 \iint\left(s(\Delta \varphi)|\nabla \psi|^{2}-s^{3}(\Delta \varphi)|\nabla \varphi|^{2}|\psi|^{2}\right) \\
& \quad=\left\|w_{s}-(s(\Delta \varphi)-\widetilde{a}) \psi\right\|^{2}+4 s \iint\left(\partial_{i} \partial_{j} \varphi\right) \partial_{i} \psi \partial_{j} \psi \\
& \quad+s \iint \partial_{t}^{2} \varphi|\psi|^{2}+2 s^{2} \iint\left(2 \partial_{i} \partial_{t} \varphi \partial_{i} \varphi+\left(\partial_{t} \varphi\right) \Delta \varphi\right)|\psi|^{2}
\end{aligned}
$$

Using the particular form of $\varphi$ we see that, for some $\lambda_{1}$ and for all $\lambda \geq \lambda_{1}$, one must have

$$
\begin{aligned}
& \left(-\partial_{i} \partial_{j} \varphi\right) \partial_{i} \varphi \partial_{j} \varphi=e^{3 \lambda \rho^{0}} \frac{\lambda^{3}}{t^{3}(T-t)^{3}}\left(\partial_{i} \partial_{j} \rho^{0}+\lambda \partial_{i} \rho^{0} \partial_{j} \rho^{0}\right) \partial_{i} \rho^{0} \partial_{j} \rho^{0} \\
& \quad \geq A \lambda|\nabla \varphi|^{3}+A|\nabla \varphi|^{3}
\end{aligned}
$$

(here, $A$ does not depend on $T$ ). We also see that

$$
\left|\partial_{t}^{2} \varphi\right|+\left|\partial_{i} \partial_{t} \varphi \partial_{i} \varphi\right|+\left|\partial_{t} \varphi \Delta \varphi\right|+\left|\Delta^{2} \varphi\right| \leq C|\nabla \varphi|^{3} .
$$


Consequently,

$$
\begin{aligned}
& +s \iint \partial_{t}^{2} \varphi|\psi|^{2}+2 s^{2} \iint\left(2 \partial_{i} \partial_{t} \varphi \partial_{i} \varphi+\partial_{t} \varphi \Delta \varphi\right)|\psi|^{2} \\
& \quad \leq C s^{2} \iint|\nabla \varphi|^{3}|\psi|^{2}
\end{aligned}
$$

and the last two terms in (6.3) can be controlled by the third term in the left for $s$ large enough. This and the fact that $\partial_{n} \varphi \geq 0$ on $\widetilde{\Sigma}_{0}$ lead to the following for $\lambda \geq \lambda_{1}$ and $s \geq s_{1}+s_{2}\|a\|_{Y}^{2 / 3}$ :

$$
\begin{aligned}
& \left\|M_{1} \psi\right\|^{2}+\left\|M_{2} \psi\right\|^{2} \\
& \quad+A s^{3} \lambda \iint|\nabla \varphi|^{3}|\psi|^{2}+A s^{3} \iint|\nabla \varphi|^{3}|\psi|^{2} \\
& \quad \leq\left\|w_{s}\right\|^{2}+4 s \iint\left(\partial_{i} \partial_{j} \varphi\right) \partial_{i} \psi \partial_{j} \psi \\
& \quad-2 \iint\left(s(\Delta \varphi)|\nabla \psi|^{2}-s^{3}(\Delta \varphi)|\nabla \varphi|^{2}|\psi|^{2}\right) .
\end{aligned}
$$

On the other hand, from (6.1) we notice that

$$
\begin{aligned}
J & =\iint\left(s(\Delta \varphi)|\nabla \psi|^{2}-s^{3}(\Delta \varphi)|\nabla \varphi|^{2}|\psi|^{2}\right) \\
& =\frac{s}{2} \iint\left(\Delta^{2} \varphi\right)|\psi|^{2}+s^{2} \iint \partial_{t} \varphi(\Delta \varphi)|\psi|^{2} \\
& +s \iint\left(M_{1} \psi-w_{s}+(s(\Delta \varphi)-\widetilde{a}) \psi\right)(\Delta \varphi) \psi,
\end{aligned}
$$

whence

$$
|J| \leq \frac{1}{2}\left\|M_{1} \psi\right\|^{2}+\left\|w_{s}\right\|^{2}+C \iint\left(|\widetilde{a}|^{2}+s\left|\Delta^{2} \varphi\right|+s^{2}|\nabla \varphi|^{3}+s^{2}|\Delta \varphi|^{2}\right)|\psi|^{2} .
$$

Using again (6.4), one obtains the following inequalities, which must hold for all $s \geq s_{3}+s_{4}\|a\|_{Y}^{2 / 3}$ :

$$
\begin{aligned}
& \left\|M_{1} \psi\right\|^{2}+\left\|M_{2} \psi\right\|^{2} \\
& \quad+A s^{3} \lambda \iint|\nabla \varphi|^{3}|\psi|^{2}+A s^{3} \iint|\nabla \varphi|^{3}|\psi|^{2} \\
& \quad \leq C\left\|w_{s}\right\|^{2}+4 s \iint\left(\partial_{i} \partial_{j} \varphi\right) \partial_{i} \psi \partial_{j} \psi .
\end{aligned}
$$

There exists $s_{5}$ such that

$$
\left\|M_{2} \psi\right\|^{2}+A s^{3} \iint|\nabla \varphi|^{3}|\psi|^{2} \geq \frac{A}{s} \iint|\nabla \varphi|^{-1}|\Delta \psi|^{2}
$$

for all $s \geq s_{5}$. Indeed, one has:

$$
\begin{aligned}
& \frac{1}{s} \iint|\nabla \varphi|^{-1}|\Delta \psi|^{2} \\
& \quad=\frac{1}{s} \iint|\nabla \varphi|^{-1}\left(M_{2} \psi-\left(s^{2}|\nabla \varphi|^{2} \psi+s \partial_{t} \varphi \psi\right)\right)^{2} \\
& \quad \leq\left\|M_{2} \psi\right\|^{2}+C s^{3} \iint|\nabla \varphi|^{3}|\psi|^{2} .
\end{aligned}
$$


Also,

$$
\frac{1}{s} \iint|\nabla \varphi|^{-1}|\Delta \psi|^{2}+s^{3} \lambda \iint|\nabla \varphi|^{3}|\psi|^{2} \geq A s \lambda^{1 / 2} \iint|\nabla \varphi| \cdot|\nabla \psi|^{2}
$$

for all $s \geq s_{6}$ for some $s_{6} \geq s_{5}$. This can be seen as follows:

$$
\begin{aligned}
& s \lambda^{1 / 2} \iint|\nabla \varphi||\nabla \psi|^{2} \\
& \quad=s \lambda^{1 / 2} \iint|\nabla \varphi|(-\Delta \psi) \psi-\frac{1}{2} s \lambda^{1 / 2} \iint \nabla|\nabla \varphi| \cdot \nabla\left(\psi^{2}\right) \\
& \quad \leq \frac{1}{2 s} \iint|\nabla \varphi|^{-1}|\Delta \psi|^{2}+\frac{1}{2} s^{3} \lambda \iint|\nabla \varphi|^{3}|\psi|^{2}+\frac{1}{2} s \lambda^{1 / 2} \iint(\Delta|\nabla \varphi|)|\psi|^{2} .
\end{aligned}
$$

From $(6.5),(6.6)$ and $(6.7)$, we see that

$$
\begin{aligned}
& \left\|M_{1} \psi\right\|^{2}+\frac{1}{s} \iint|\nabla \varphi|^{-1}|\Delta \psi|^{2} \\
& +s \lambda^{1 / 2} \iint|\nabla \varphi||\nabla \psi|^{2}+s^{3} \lambda \iint|\nabla \varphi|^{3}|\psi|^{2} \\
& \quad \leq C\left\|w_{s}\right\|^{2}+C s \iint\left(\partial_{i} \partial_{j} \varphi\right) \partial_{i} \psi \partial_{j} \psi
\end{aligned}
$$

for all $s \geq s_{6}+s_{4}\|a\|_{Y}^{2 / 3}$ and all $\lambda \geq \lambda_{1}$. We can thus choose (and fix) $\lambda$ sufficiently large (depending on $G, S_{1}$ and $T$ ) to control the last term in the right with the fourth term in the left. After this, we can write

$$
\begin{aligned}
& \left\|M_{1} \psi\right\|^{2}+\frac{1}{s} \iint|\nabla \varphi|^{-1}|\Delta \psi|^{2} \\
& \quad+s \iint|\nabla \varphi||\nabla \psi|^{2}+s^{3} \iint|\nabla \varphi|^{3}|\psi|^{2} \\
& \quad \leq C\left\|w_{s}\right\|^{2} .
\end{aligned}
$$

It is also true that, for some $s_{7} \geq s_{6}$ and for all $s \geq s_{7}$,

$$
\left\|M_{1} \psi\right\|^{2}+s \iint|\nabla \varphi||\nabla \psi|^{2} \geq \frac{A}{s} \iint|\nabla \varphi|^{-1}\left|\partial_{t} \psi\right|^{2} .
$$

Indeed, when $s$ is sufficiently large, one has:

$$
\begin{aligned}
& \frac{1}{s} \iint|\nabla \varphi|^{-1}\left|\partial_{t} \psi\right|^{2} \\
& \quad=\frac{1}{s} \iint|\nabla \varphi|^{-1}\left(M_{1} \psi-2 s \nabla \varphi \cdot \nabla \psi\right)^{2} \\
& \quad \leq\left\|M_{1} \psi\right\|^{2}+C s \iint|\nabla \varphi|^{3}|\psi|^{2}
\end{aligned}
$$

Consequently,

$$
\begin{aligned}
& \frac{1}{s} \iint|\nabla \varphi|^{-1}\left(\left|\partial_{t} \psi\right|^{2}+|\Delta \psi|^{2}\right) \\
& \quad+s \iint|\nabla \varphi||\nabla \psi|^{2}+s^{3} \iint|\nabla \varphi|^{3}|\psi|^{2} \\
& \quad \leq C\left\|w_{s}\right\|^{2}
\end{aligned}
$$


for all $s \geq s_{7}+s_{4}\|a\|_{Y}^{2 / 3}$. Replacing $\psi$ by $\rho^{-s} q$ in $(6.8)$, it is not difficult to deduce (5.1) for $s \geq s_{8}+s_{4}\|a\|_{Y}^{2 / 3}$. Hence, lemma 5.2 is proved.

The author is indebted to J.-P. Puel and E. Zuazua for several fruitful discussions.

\section{REFERENCES}

[1] D. Chae, O.Yu. Imanuvilov and S.M. Kim: Exact controllability for semilinear parabolic equations with Neumann boundary conditions, to appear.

[2] C. Fabre, J.P. Puel and E. Zuazua: Approximate controllability of the semilinear heat equation, Proc. Royal Soc. Edinbourgh, 125 A, 1995, 31-61.

[3] A.V. Fursikov and O.Yu. Imanuvilov: On controllability of certain systems simulating a fluid flow, in Flow Control, IMA Vol. Math. Appl., 68, Ed. by M.D. Gunzburger, Springer-Verlag, New York, 1995.

[4] A.V. Fursikov and O.Yu. Imanuvilov: Local exact controllability for 2-D NavierStokes equations, to appear.

[5] O.Yu. Imanuvilov: Thesis, Moscow 1991 (in russian); see also: Exact boundary controllability of the parabolic equation, Russian Math. Surveys, 48, No. 3, 1993, 211212.

[6] O.Yu. Imanuvilov: Boundary controllability of parabolic equations, Russian Acad. Sci. Sb. Math., 186, No. 6, 1995, 109-132.

[7] O.A. Ladyzhenskaja, V.A. Solonnikov and N.N. Ural'ceva: Linear and quasilinear equations of parabolic type, Trans. Math. Monographs, Vol. 23, AMS, Providence, 1968.

[8] G. Lebeau and L. Robbiano: Contrôle exact de l'équation de la chaleur, Comm. P.D.E., 20, 1995, 335-356.

[9] G. Lebeau and E. Zuazua: Null controllability of a system of linear thermoelasticity, Arch. Rat. Mech. Anal., to appear.

[10] E. Zuazua: Exact boundary controllability for the semilinear wave equation, in Nonlinear differential equations and their applications, H. Brezis and J.L. Lions eds, Research Notes in Math. 10, Pitman, London, 1991, 357-391.

[11] E. Zuazua: Exact controllability for semilinear wave equations in one space dimension, Ann. Inst. Henri Poincaré, Vol. 10, No. 1, 1993, 109-129. 